\title{
Water Dimers in the Atmosphere III: Equilibrium Constant from a Flexible Potential ${ }^{\dagger}$
}

\author{
Yohann Scribano, ${ }^{\ddagger}$ Nir Goldman, ${ }^{\S}$ R. J. Saykally," and Claude Leforestier*, \\ UMR-CNRS 5636, CC 014, Université Montpellier II, 34095 Montpellier, France, Lawrence Livermore \\ National Laboratory, L-268, Livermore, California 94550, and Department of Chemistry, University of \\ California, Berkeley, California 94720-1460
}

Received: November 22, 2005; In Final Form: February 10, 2006

\begin{abstract}
We present new results for the water dimer equilibrium constant $K_{\mathrm{p}}(T)$ in the range 190-390 K, using a flexible potential energy surface fitted to spectroscopical data. The increased numerical complexity due to explicit consideration of the monomer vibrations is handled via an adiabatic $(6+6) d$ decoupling between intra- and intermolecular modes. The convergence of the canonical partition function of the dimer is ensured by computing all energy levels up to dissociation for total angular momentum values $J=0-5$ and using an extrapolation scheme to higher values. The newly calculated values for $K_{\mathrm{p}}(T)$ are in very good agreement with available experimental data at room temperature. At higher temperatures, an analysis of the convergence of the partition function reveals that quasi-bound states are likely to contribute to the equilibrium constant. Additional thermodynamical quantities $\left(\Delta G, \Delta H, \Delta S\right.$, and $\left.C_{\mathrm{p}}\right)$ have also been determined and fit to quadratic expressions $a+b T+c T^{2}$.
\end{abstract}

\section{Introduction}

In recent years, it has been proposed that the water dimers may be important in several atmospheric processes, such as excess absorption of solar radiation, ${ }^{1-5}$ water continuum absorption in the far infrared, ${ }^{6,7}$ homogeneous nucleation of water into droplets and ice, ${ }^{8,9}$ and catalysis of important chemical reactions, ${ }^{10,11}$ such as acid rain formation. However, from a quantitative viewpoint, its influence remains a controversial issue, as its concentration and variation with conditions have not yet been precisely established.

For a long time, the only available values for the dimerization constant $K_{\mathrm{p}}(T)$ were from the thermal conductivity of the classic steam experiments of Curtiss et al., ${ }^{12}$ which provided results only over a very limited temperature range of $358-386 \mathrm{~K}$. Another source of experimental data comes from the measurement of the second virial coefficient $B(T)$ through the relation ${ }^{12}$ $K_{\mathrm{p}}(T) \simeq-\left(B-b_{0}\right) / R T$.

Recently, different experiments aimed at assessing dimer concentrations at lower temperatures, more relevant to atmospheric processes, have appeared. (i) A direct measurement was attempted by Pfeilsticker et al. ${ }^{13}$ by means of atmospheric long path absorption of the third overtone $|0\rangle_{f}|4\rangle_{b}$ of the $\mathrm{OH}$ stretching mode. The resulting value for $K_{\mathrm{p}}$, obtained at a unique temperature $(292 \mathrm{~K})$, was determined using the water dimer line strength calculated by Low and Kjaergaard. ${ }^{14}$ However, recent cavity ringdown measurements ${ }^{15}$ of the water monomer absorption in the same spectral region revealed 123 new lines, which seems to question the validity of the interpretation of the dimer spectrum by Pfeilsticker et al. (ii) Ptashnik et al. ${ }^{16}$ obtained highspectral-resolution pure water vapor absorption spectra for two different temperatures (299 and $342 \mathrm{~K}$ ). Using HITRAN database and two different representations of the water con-

\footnotetext{
† Part of the special issue "John C. Light Festschrift".

* Corresponding author. E-mail: Claude.Leforestier@univ-montp2.fr.

† Université Montpellier II.

$\S$ Lawrence Livermore National Laboratory.

"University of California.
}

tinuum (Clough-Kneizys-Davies and Ma-Tipping), they derived $K_{\mathrm{p}}$ values from a fit of the residual.

In two previous papers, ${ }^{17,18}$ we presented a theoretical determination of the dimerization constant $K_{\mathrm{p}}(T)$ via a calculation of the canonical water dimer partition function. The rovibrational energy levels were computed on the VRT-ASP dimer potential energy surfaces previously fitted to $\left(\mathrm{D}_{2} \mathrm{O}\right)_{2}$ microwave and far-infrared transitions. These surfaces consider the monomer to be rigid, i.e., constrained to some reference geometry over the potential well up to dissociation. In these calculations, because of limited computing resources available at that time, only the $J=0$ levels were actually computed. The $J>0$ values were extrapolated using the symmetric top energy level pattern.

In the present work, we improve these calculations in several ways:

(i) We employ a flexible potential energy surface fitted to known $\left(\mathrm{H}_{2} \mathrm{O}\right)_{2}$ transitions. ${ }^{19}$ Flexibility allows the water dimer to change its monomer geometries with intermolecular separation, and thus provides a more accurate description of excited dimer energy levels.

(ii) A larger basis set is used, resulting in an increased number of bound states. Notably, because of its weak binding energy, the bound energy levels located in the highest $100 \mathrm{~cm}^{-1}$ contribute for ca. $5 \%$ of the partition function at $300 \mathrm{~K}$.

(iii) All the bound states, up to dissociation, were computed for $J$ values of $0,1, \ldots, 5(K=0, \ldots, J)$. As the calculated splittings vary substantially with the $K$ quantum number, the extrapolation scheme is only required for $K>5$ values for which those splittings stabilize.

The outline of this paper is as follows. We first briefly recall our $(6+6) d$ adiabatic treatment of the system and the potential energy surface considered. We then present the bound states calculations performed and the scheme used to extrapolate the calculated energy levels to high $J$ values. This scheme allows us to compute the dimer partition function and to determine the equilibrium constant in the range $250-400 \mathrm{~K}$, which is 
compared to available experimental values. From the partition function, we can also evaluate the temperature dependence of different thermodynamic properties of the dimer. We conclude by discussing some future directions to be investigated.

\section{Bound States Calculations}

In this section, we briefly recall the $(6+6) d$ adiabatic approximation that we previously developed in order to explicitly deal with the flexibility of the monomers (see ref 19 for a detailed description). The method employed to obtain converged eigenstates (Lanczos recursive scheme) in ultralarge basis sets is then discussed. Using the VRT(MCY-5f) flexible potential previously fitted to experimental results, ${ }^{19}$ we first consider the energy levels pattern in order to justify the symmetric top approximation used later on. We then discuss the variation of the acceptor tunneling splitting as a function of the $J$ and $K$ quantum numbers. The observed behavior allows us to define an extrapolation scheme to very high $J$ values, which are needed in order to compute the equilibrium constant.

A. $(6+6) d$ Adiabatic Approximation. In the rigid approximation for the monomers, the molecular Hamiltonian is ${ }^{20,21}$

$$
\begin{aligned}
& \mathbf{H}_{\text {rigid }}=- \frac{\hbar^{2}}{2 \mu_{\mathrm{AB}}} \frac{1}{\mathscr{R}} \frac{\partial^{2}}{\partial \mathscr{R}^{2}} \mathscr{R}+\mathbf{H}_{\mathrm{rot}}^{\mathrm{A}}+\mathbf{H}_{\mathrm{rot}}^{\mathrm{B}}+ \\
& \mathbf{V}\left(\mathscr{R}, \Omega^{\mathrm{A}}, \Omega^{\mathrm{B}}\right)+\frac{1}{2 \mu_{\mathrm{AB}} \mathscr{R}^{2}}\left\{\mathbf{J}^{2}+\mathbf{j}^{2}-2 \mathbf{j} \cdot \mathbf{J}\right\}
\end{aligned}
$$

where

- $R$ is the distance between the centers of mass of the two monomers $\mathrm{A}$ and $\mathrm{B}$, and $\mu_{\mathrm{AB}}$ their reduced mass.

- $\mathbf{H}_{\text {rot }}^{\mathrm{X}}$ and $\mathbf{j}^{\mathrm{X}}$ are respectively the rigid rotational Hamiltonian and total angular momentum of monomer $\mathrm{X}(\mathrm{X}=\mathrm{A}, \mathrm{B})$.

- $\mathbf{j}=\mathbf{j}^{\mathrm{A}}+\mathbf{j}^{\mathrm{B}}$ is the coupled internal rotational angular momentum.

- $\mathbf{J}=\mathbf{j}+\mathbf{L}$ is the total angular momentum ( $\mathbf{L}$ is the relative angular momentum between the monomer centers of mass).

- $\Omega^{\mathrm{X}} \equiv\left(\varphi^{\mathrm{X}}, \theta^{\mathrm{X}}, \chi^{\mathrm{X}}\right)$ represents the Euler angles defining the orientation of monomer $\mathrm{X}$ in the dimer body fixed (BF) frame.

To deal with the flexibility of the monomers, we replace in eq 1 the above rigid potential $\mathbf{V}\left(R, \Omega^{\mathrm{A}}, \Omega^{\mathrm{B}}\right)$ by its adiabatic counterpart $\mathbf{V}_{\mathrm{ad}}\left(\mathscr{R}, \Omega^{\mathrm{A}}, \Omega^{\mathrm{B}}\right)$ which takes into account the variation of the intramolecular zero-point energy as a function of the intermolecular geometry. More specifically, at each point of the six-dimensional grid associated to the intermolecular coordinates, we solve for the ground-state energy of the intramolecular modes using the successive adiabatic reduction method of Bačić and Light. ${ }^{22,23}$ The resulting Hamiltonian operator will be denoted by $\mathbf{H}$ in the following. Such a $(6+$ 6) $d$ adiabatic approximation is justified by the high frequencies of these modes as compared to the intermolecular ones. In this approximation, we also consider the intermolecular geometry dependence of each monomer rotational constants $B_{x}, B_{y}$, and $B_{z}$ (entering the definition of the $\mathbf{H}_{\mathrm{rot}}^{\mathrm{A}}+\mathbf{H}_{\mathrm{rot}}^{\mathrm{B}}$ operators). This adiabatic approximation allows us to recast the flexible monomers calculation into a rigid one, which was shown to be efficiently handled by the pseudo-spectral split Hamiltonian method. ${ }^{24,25}$

B. Lanczos Calculation of Bound States. To compute the equilibrium constant of the dimer in the 250-400 K temperature range, we have to consider all excited rovibrational states up to dissociation and high $J$ values of the total angular momentum.
This results in a huge number of molecular basis states required to converge the required energy levels. An obvious way to reduce this number is to separately perform the calculations for each irreducible representation (IR) of the permutationinversion group $\mathrm{G}_{16}$ of the dimer. ${ }^{26}$ Second, as the symmetryadapted bases are still much too large (over $10^{6}$ states), diagonalization was performed by the standard Lanczos algorithm ${ }^{27}$ without reorthogonalization.

The actual bound states calculation was performed in two steps:

(i) In the first one, one builds the Krylov space $\mathscr{K}=$ $\left\{\left|u_{n}\right\rangle\right\}_{n=0}^{N}$

$$
\beta_{n+1}\left|u_{n+1}\right\rangle=\left(\mathbf{H}-T_{n, n}\right)\left|u_{n}\right\rangle-T_{n, n+1}\left|u_{n-1}\right\rangle
$$

by repetitive actions of the Hamiltonian operator on a seed vector $\left|u_{0}\right\rangle$. Diagonalization of the resulting tridiagonal Hamiltonian matrix $\boldsymbol{T}$ (of size 3000 at most) leads to the eigenstates expressed in the Krylov space:

$$
\left|\Psi_{\alpha}\right\rangle=\sum_{n=0}^{N} C_{n \alpha}\left|u_{n}\right\rangle
$$

(ii) In a second step, one performs an identical Lanczos scheme using the same seed vector $\left|u_{0}\right\rangle$ and generates on the fly the eigenstates expressed in the initial molecular basis set

$$
\left|\Psi_{\alpha}\right\rangle:=\left|\Psi_{\alpha}\right\rangle+C_{n \alpha}\left|u_{n}\right\rangle \quad(n=0, \ldots, N)
$$

each time a new Lanczos vector $\left|u_{n}\right\rangle$ is produced. This twostep procedure is required, as the molecular basis size is too large for the Lanczos vectors to be stored during the first pass. For the system considered here, 3000 recursions typically ensures convergence of the lowest 150 bound states at a fixed total angular momentum value $J$ and for a given IR. Care must be taken in removing ghosts and spurious eigenvalues ${ }^{28}$ from the list obtained by diagonalization in the Krylov space.

The Lanczos scheme can also be used to compute all the energy levels up to dissociation. If one is only interested in the energies, and not in the associated eigenstates, one can let step (i) run for a very large number of iterations (up to 70000 in the present case) until all the bound energies are converged. The modified QL algorithm of Wyatt and Scott ${ }^{29}$ was used when only the eigenvalues were needed. Identification of spurious eigenvalues can be efficiently realized by computing the error norm

$$
\sigma_{\alpha}=\left\|\left(\mathbf{H}-E_{\alpha}\right) \Psi_{\alpha}\right\|
$$

without actually requiring the knowledge of the eigenstates $\Psi_{\alpha}$ but using a minor extension of the modified QL algorithm. ${ }^{30}$

C. Potential Energy Surface. In the calculations presented here, we used the flexible VRT-MCYf potential energy surface previously fitted to experiments ${ }^{19}$

$$
\begin{aligned}
\mathbf{V}\left(\mathbf{q}^{\mathrm{A}}, \mathbf{q}^{\mathrm{B}}, \mathscr{R}, \Omega^{\mathrm{A}}, \Omega^{\mathrm{B}}\right)=V_{\mathrm{JPT} 2}\left(\mathbf{q}^{\mathrm{A}}\right)+V_{\mathrm{JPT} 2}\left(\mathbf{q}^{\mathrm{B}}\right)+ \\
V^{(2)}\left(\mathbf{q}^{\mathrm{A}}, \mathbf{q}^{\mathrm{B}}, \mathscr{R}, \Omega^{\mathrm{A}}, \Omega^{\mathrm{B}}\right)
\end{aligned}
$$

In the above expression, $\mathbf{q}^{\mathrm{X}}$ stands for the three internal coordinates of monomer $\mathrm{X}$, and $V_{\mathrm{JPT} 2}$ refers to the JPT2 water potential energy surface as fitted by Tennyson and collaborators. ${ }^{31}$ Only the two-body part $V^{(2)}(\ldots)$ was fitted to known microwave and far-infrared transitions. It must be recalled that the water dimer corresponds to a very flexible molecule, far from the harmonic oscillator model. As a consequence, the zero- 


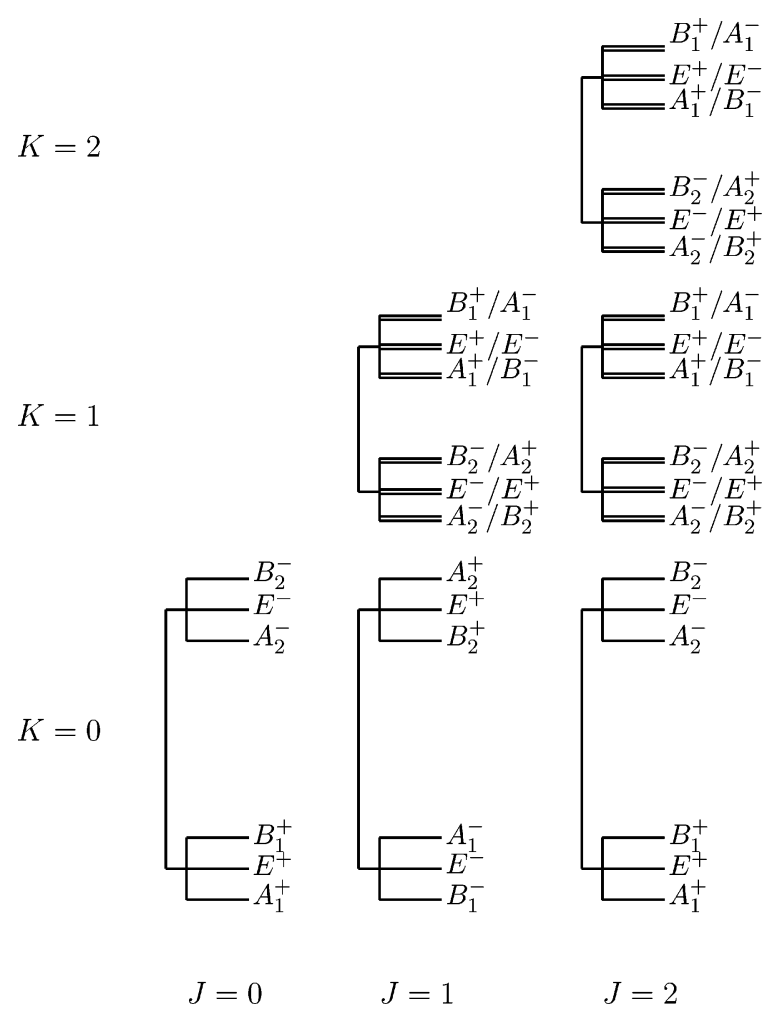

Figure 1. Rotational energy levels pattern of $\mathrm{A}^{\prime}$ states associated to a given $v$ vibrational state. Each fork corresponds to levels split by tunneling between the eight equivalent minima of the potential energy surface. The quasi degeneracy observed for $K>0$ components results from the water dimer being a nearly symmetric top.

point energy $\left(\sim 600 \mathrm{~cm}^{-1}\right)$ represents a significant fraction of the binding energy $\left(D_{\mathrm{e}} \approx 1750 \mathrm{~cm}^{-1}\right)$. The set of transitions considered (up to $160 \mathrm{~cm}^{-1}$ of excitation energy) thus allows the fitting procedure to sample the lower half of the potential well. In the fit, the binding energy $D_{\mathrm{e}}$ was also constrained to the best converged theoretical value of $21 \mathrm{~kJ} / \mathrm{mol}^{32-35}$ available at that time. It results in a dissociation energy value $D_{0} \approx 1230$ $\mathrm{cm}^{-1}$, to be compared with the value $1070 \mathrm{~cm}^{-1}$ associated to the rigid VRT(ASP-W)III potential used in our previous calculations. ${ }^{18}$

As the two-body term vanishes at large separation, $\mathscr{R}$, flexibility enforces the correct (free equilibrium) geometry for the monomers near the dissociation limit. This effect is particularly important for the $\mathrm{OH}$ distance involved in the $\mathrm{O}-\mathrm{H} \cdots \mathrm{O}$ hydrogen bond, which changes from $0.968 \AA$ at the equilibrium geometry of the dimer to the free equilibrium value $0.958 \AA$. Although this effect might appear small, the zeropoint energy of the donor molecule changes accordingly from $4569 \mathrm{~cm}^{-1}$ to $4634 \mathrm{~cm}^{-1}$.

All the calculations presented in this work have been performed with the same basis set as that used for fitting the flexible dimer potential, ${ }^{19}$ namely, (i) a Wigner basis set up to $j=10$ on each monomer and (ii) an initial radial basis set of 16 sine functions spanning the box $4.2 \leq R \leq 10$ (bohr), contracted to 9 grid points by means of the HEG procedure. ${ }^{36,37}$

D. Energy Level Patterns. For different $K>0$ values up to $J=5$, we have computed the energy levels associated to different IRs. It appears that all these levels are quasi-doubly degenerate according to the following associations

$$
\left\{\mathrm{A}_{1}^{ \pm} / \mathrm{B}_{1}^{\mp}\right\},\left\{\mathrm{A}_{2}^{ \pm} / \mathrm{B}_{2}^{\mp}\right\},\left\{\mathrm{E}^{+} / \mathrm{E}^{-}\right\}
$$

exemplified in Figure 1 (actually, the $\mathrm{B}_{1}^{ \pm}$energy levels do not exist because of zero nuclear spin statistics). This results from the dimer being a quasi-symmetric top, as shown by the values (in $\mathrm{cm}^{-1}$ ) of the rotational constants of the ground state

$$
A=7.858, \quad \frac{1}{2}(B+C)=0.187, \quad \frac{1}{2}(B-C) \simeq 3 \times 10^{-4}
$$

which leads to a symmetry parameter value $\kappa=-0.9998$ (prolate top). Henceforth, in our calculations, we will resort to a symmetric top approximation which consists of neglecting the $2 \mathbf{j} \cdot \mathbf{J}$ term in the expression (eq 1) of the Hamiltonian.

As we are interested in computing the dimer spectrum at room temperature, very high $J$ values will be needed in order to converge the rotational partition function. For such high $J$ values, the bound states calculations cannot be performed, and one has to resort to some extrapolation scheme, which will be presented in the next section. The frequently used $J$-shifting approximation $^{38,39}$ is essentially a molecular version of the wavenumber approximation of atomic physics. It considers the vibration and rotation to be decoupled and expresses the vibration-rotation energy $E_{v J K}$ as that of a prolate top

$$
E_{v J K}=E_{v}+B J(J+1)+(A-B) K^{2}
$$

in the case considered here. Such an approximation cannot be directly used here, as each level is split into six sublevels due to tunneling effects (see Figure 1). Furthermore, these splittings strongly depend on the $K$ value. Figure 2 displays both the acceptor and donor-acceptor splittings for the ground $(v=0)$ state, as a function of $J$ and $K$, up to $J=5$.

One can see from this figure that the acceptor splitting values (boldface figures) strongly depend on $K$ in the bottom of the spectrum and then tend to stabilize at higher values of $K$. The donor-acceptor splittings show a similar pattern, although the variation is smoother. However, both splittings do not depend on $J$ for a fixed $K$ value. Despite the strong variation of these splitting values, one can make use of the relation given by eq 6 for the center of each fork, defined as

$$
\bar{E}_{v J K}=\frac{1}{4}\left[E\left(\mathrm{~A}_{i}^{+}\right)+E\left(\mathrm{~B}_{i}^{+}\right)+E\left(\mathrm{~A}_{j}^{-}\right)+E\left(\mathrm{~A}_{j}^{-}\right)\right]
$$

where the $(i, j)$ indices can take the values $(1,2)$ or $(2,1)$. In Figure 2, the numbers in brackets on the $K=0$ row report the difference $\bar{E}_{v J 0}-\bar{E}_{v 00}$, which obeys the $B J(J+1)$ law. Similarly, the numbers in parentheses appearing on the $K>0$ rows report the difference $\bar{E}_{v J K}-E_{v J 0}$ which follows the (A$B) K^{2}$ law. Although only displayed for the ground state, one finds the same pattern for all vibrational states.

E. Extrapolation to High $\boldsymbol{J}$ Values. To build a data basis for energy extrapolation to high $J$ values, all the bound energy levels up to dissociation were calculated for all IRs, for $J$ values $0,1, \ldots, 5$, and for associated $K$ values $0,1, \ldots, J$. Actually, only the $\mathrm{A}_{1}^{+}, \mathrm{A}_{1}^{-}, \mathrm{A}_{2}^{+}, \mathrm{A}_{2}^{-}$, and $\mathrm{E}^{+}$representations were considered for $K>0$ values. Because of the symmetric top approximation previously invoked, the $\mathrm{B}_{2}^{+}, \mathrm{B}_{2}^{-}$, and $\mathrm{E}^{-}$energy levels are degenerate with the $\mathrm{A}_{2}^{-}, \mathrm{A}_{2}^{+}$, and $\mathrm{E}^{+}$, respectively. One can then estimate the energy of an arbitrary $(v, J, K, \Gamma)$ energy level according to a modified $J$-shifting approximation

$$
\begin{aligned}
E_{v J K}^{\Gamma}=E_{v J_{\mathrm{o}} K_{\mathrm{o}}}^{\Gamma}+B_{v J K}^{\Gamma} J(J+1)-B_{v J_{\mathrm{o}} K_{\mathrm{o}}}^{\Gamma} J_{\mathrm{o}}\left(J_{\mathrm{o}}+1\right)+ \\
\left(A_{v J K}^{\Gamma}-B_{v J K}^{\Gamma}\right) K^{2}-\left(A_{v J_{\mathrm{o}} K_{\mathrm{o}}}^{\Gamma}-B_{v J_{\mathrm{o}} K_{\mathrm{o}}}^{\Gamma} K_{\mathrm{o}}^{2}\right.
\end{aligned}
$$


$\mathrm{K}=5$

$\mathrm{K}=4$

$\mathrm{K}=3$

\begin{tabular}{|c|c|}
\hline$\sqrt{0.26}^{B}{ }_{2}^{-} / A_{2}^{+}$ & $\overline{0.26}^{B_{2}^{-} / A_{2}^{+}}$ \\
\hline$A_{2}^{-} / I$ & $A_{2}^{-} / B_{2}^{+}$ \\
\hline $5.7 \quad(128.8)$ & $5.7 \quad(128.8)$ \\
\hline$B_{1}^{+}$ & $\left.\right|_{{ }^{-}} B_{1}^{+} / A_{1}^{-}$ \\
\hline & 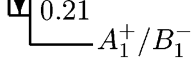 \\
\hline
\end{tabular}

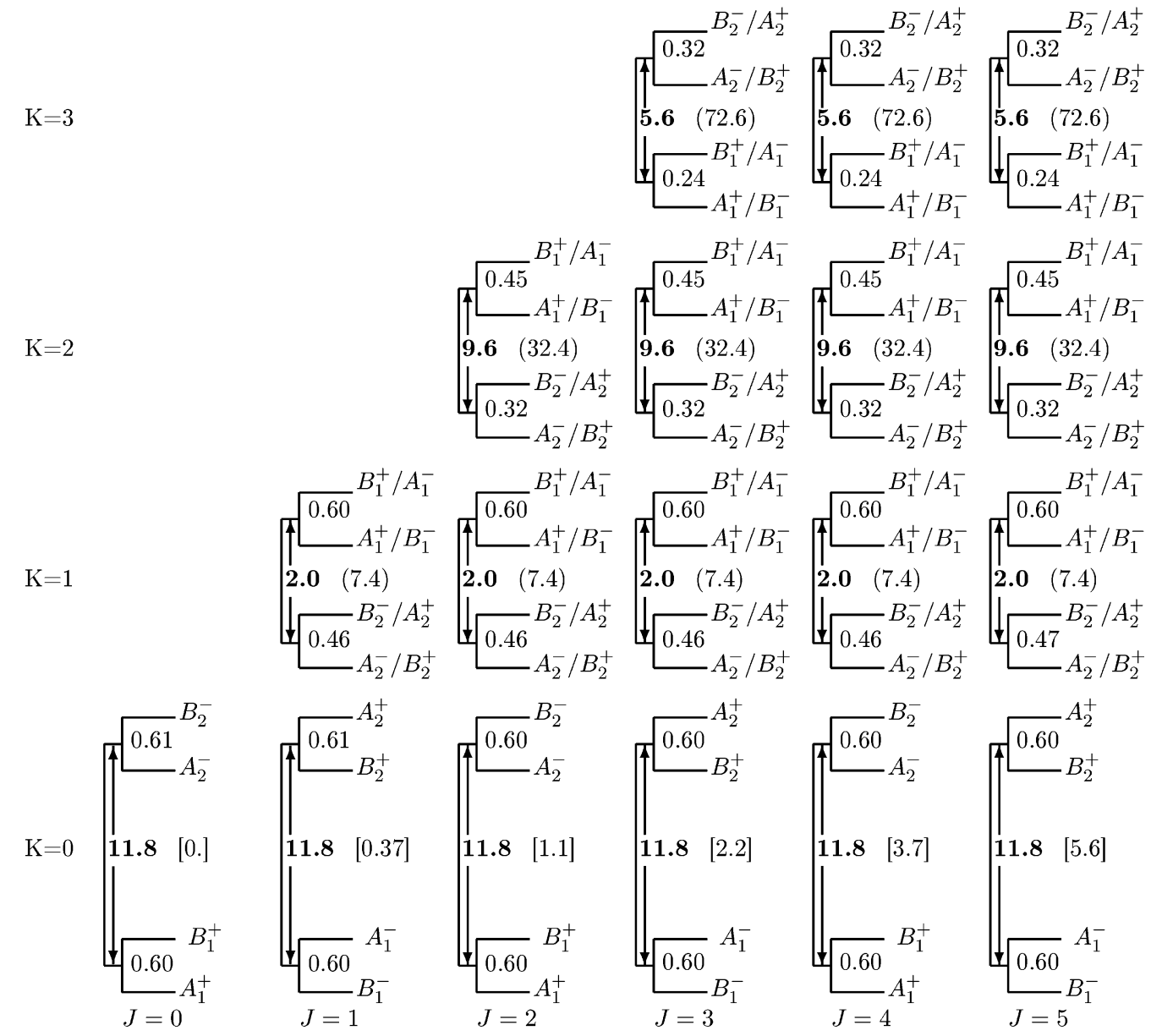

Figure 2. Representation of the ground state $(v=0)$ rotational manifold up to $J=5$. For sake of clarity, the $\mathrm{E}^{ \pm}$states are not reported. Boldface figures indicate the acceptor tunneling splittings, while donor-acceptor splittings appear between each $\mathrm{A}_{i}^{ \pm} / \mathrm{B}_{i}^{ \pm}$pair. The values in square brackets give the $\bar{E}_{v J 0}-\bar{E}_{v 00}$ energy difference between the centers of the forks (eq 7), and numbers in parentheses report the $\bar{E}_{v J K}-\bar{E}_{v J 0}$ energy change.

where $\Gamma$ stands for the IR, and $J_{\mathrm{o}}$ and $K_{\mathrm{o}}$ refer to actually calculated $\left(v, J_{0}, K_{0}, \Gamma\right)$ energy levels as shown in Figure 3. More specifically, one considers the three different situations, depending on the value of $K$ :

$K=0: J$ and $J_{\mathrm{o}}$ must be of the same parity as the IRs alternate with $J$ (see Figure 1).

$1 \leq K \leq 5$ : One then decreases the $J$-value until matching a calculated $\left(v, J_{\mathrm{o}}, K, \Gamma\right)$ level.

$K>5$ : In this case, one has to decrease both the $K$ and $J$ values according to Figure 3.

One important point to be considered concerns the a priori $(v, J, K, \Gamma)$ dependence of the $A$ and $B$ rotational constants entering eq 8 . To assess it, we calculated the lowest 150 bound states $\Psi_{v J K}^{\Gamma}$ for $\mathrm{A}_{1}^{+}, \mathrm{A}_{1}^{-}, \mathrm{A}_{2}^{+}, \mathrm{A}_{2}^{-}$, and $\mathrm{E}^{+} \mathrm{IRs}$, for
$J$ values $0,1, \ldots, 5$, and for associated $K$ values $0,1, \ldots, J$. Using the eigenfunctions, one can compute the associated rotational constants as the eigenvalues of the averaged inertia tensor

$$
\langle\mathbf{I}\rangle=\left\langle\Psi_{v J K}^{\Gamma}|\mathbf{I}| \Psi_{v J K}^{\Gamma}\right\rangle
$$

Figure 4 represents, for the $\mathrm{B}_{1}^{+}$states, the variation with $K$ of the $A$ constant and the variation with $J$ of the $B$ constant, as a function of their energies. The first plot displays the $A$-values for $J$ fixed at the value of 3 and $K$ varying from 0 to 3 , while the second one corresponds to the equivalent values for $K$ fixed at 3 and $J$ varying from 3 to 5 . 


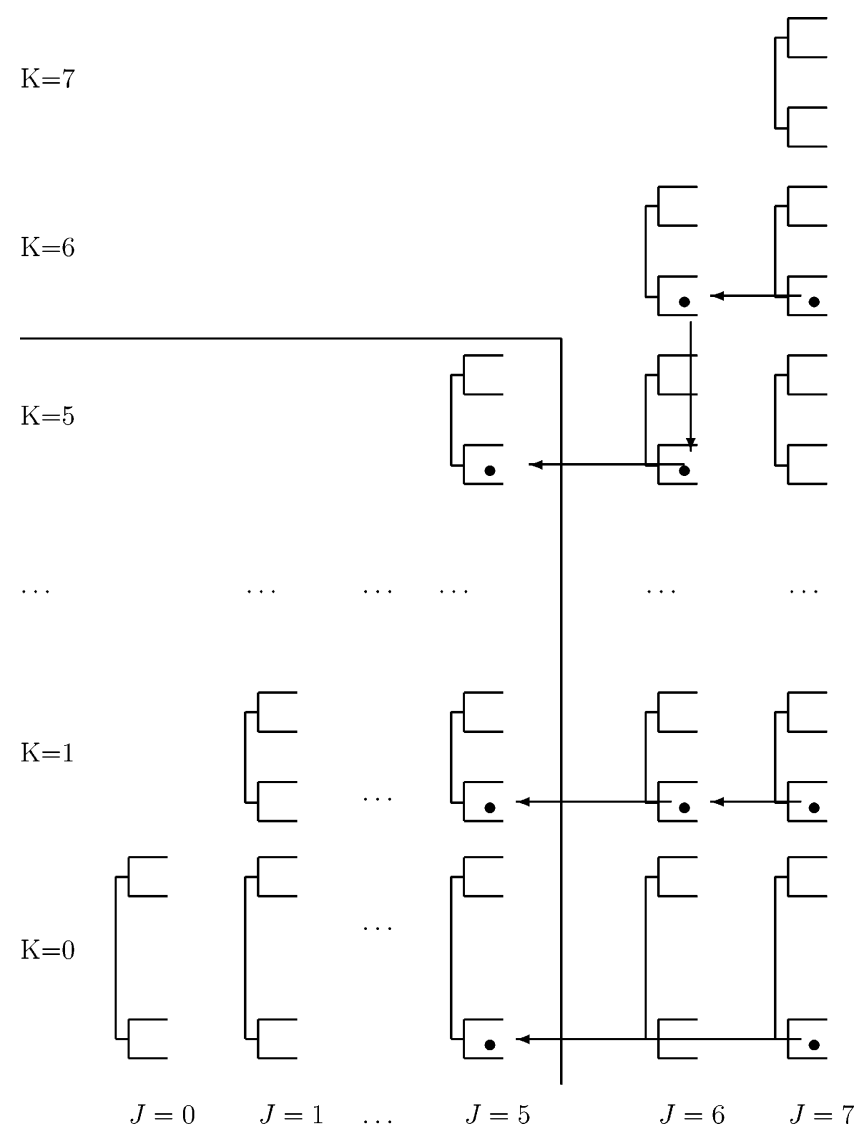

Figure 3. Scheme used to extrapolate energies to high $J$-values: All the bound states appearing in the lower left rectangle $(K \leq J \leq 5)$ have been computed and serve as $\left(J_{0}, K_{0}\right)$ reference states to be used in eq 8. The left arrows indicate the successive modifications brought to the original quantum numbers $J$ and $K$ until a reference state is reached.

Dependence With Energy. These data allowed us to perform a linear regression of the constants

$$
A_{v J K}^{\Gamma}=a_{J K}^{\Gamma}+b_{J K}^{\Gamma} E
$$

and a similar expression for $B_{v J K}^{\Gamma}$. These expressions are used to extrapolate the constants at higher energies $(v>150)$.

Dependence with $K$. It will be shown in the next section (see Table 2) that $K$-values less than or equal to 5 contribute more than $90 \%$ to the total partition function below $T=350 \mathrm{~K}$. One can then expect that using for $K>5$ the values corresponding to $K=5$

$$
A_{v J K>5}^{\Gamma} \simeq A_{v J K=5}^{\Gamma}
$$

will bring minor errors. This approximation is comforted by the fact that the averaged values (lines displayed on the $A_{J=3, K}$ vs $E$ graph) tend to coalesce at high energies.

Dependence with $J$. As high values of $J$ (up to 70) are required to converge the partition function at room temperature, one can wonder whether the centrifugal distortion changes significantly the $B$ rotational constant used in eq 8 . It will be shown in the next section (see Table 3 ) that this effect only introduces negligible errors in the evaluation of the partition function for the temperatures considered.

\section{Equilibrium Constant}

The equilibrium constant is defined from the canonical expression
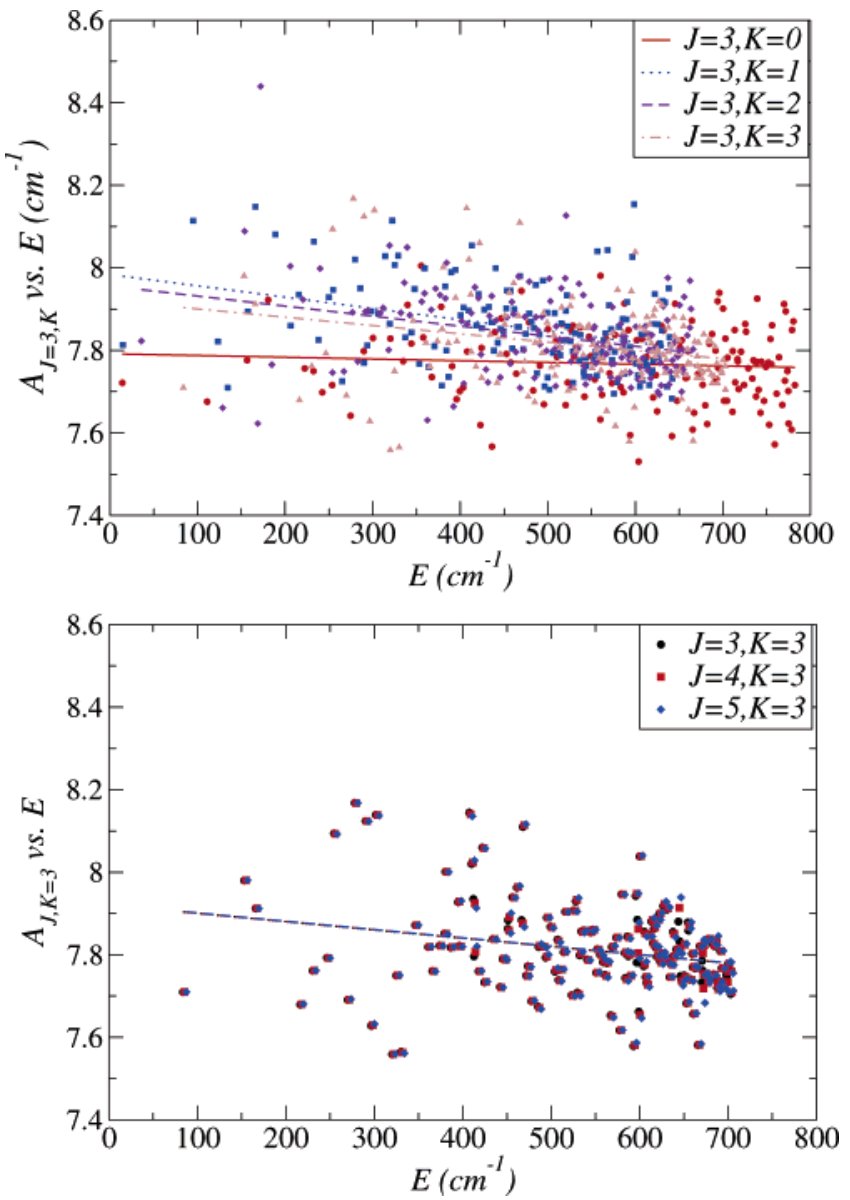

Figure 4. Variation with $J$ and $K$ of the $A$ constant for the $\mathrm{A}_{1}^{+}$ states as a function of their energies $E(v, J, K)$ : (a) $J$ fixed at the value of 3 , and $K$ varying from 0 to 3 ; (b) $K$ fixed at the value of 3, and $J$ varying from 3 to 5 . The lines correspond to a linear fit of these values.

$$
K_{\mathrm{p}}(T)=\frac{p_{\mathrm{D}}}{p_{\mathrm{M}}{ }^{2}}=\frac{1}{k T}\left(\frac{Q_{\mathrm{VR}}^{\mathrm{D}} \mathrm{e}^{D_{0} / k T}}{\lambda_{\mathrm{D}}{ }^{3}}\right)\left(\frac{Q_{\mathrm{VR}}^{\mathrm{M}}}{\lambda_{\mathrm{M}}{ }^{3}}\right)^{-2}
$$

where $\mathrm{D}$ and $\mathrm{M}$ stand, respectively, for the dimer and the monomer, and $\lambda_{\mathrm{X}}=h / \sqrt{2 \pi m_{\mathrm{X}} k T}$ represents the thermal de Broglie wavelength. The monomer rovibrational partition function $Q_{\mathrm{VR}}^{\mathrm{M}}$ was taken from Harris et al., ${ }^{40}$ who fit their calculations to a polynomial in $T$. The exponential term $\mathrm{e}^{D_{0} / k T}$ accounts for the difference in the dimer $\left(V_{0}=-D_{0}\right)$ and monomers $\left(V_{0}\right.$ $=0$ ) zeros of vibrational energies.

A. Dimer Partition Function $Q_{\mathbf{V R}}^{\mathrm{D}}$. The dimer rovibrational partition function $Q_{\mathrm{VR}}^{\mathrm{D}}$ reads as

$$
Q_{\mathrm{VR}}^{\mathrm{D}}(T)=\frac{1}{16} \sum_{\Gamma} g_{\Gamma} \sum_{v J K} g_{J} \exp \left(-E_{v J K}^{\Gamma} / k T\right)
$$

where the factors $g_{\Gamma}$, given in Table 1 , account for both space and nuclear spin degeneracies. The normalization factor $(1 / 16)$ appearing in front of eq 11 is to comply with the convention used by Harris et al. ${ }^{40}$ for the definition of the monomer partition function which affects weights of $3 / 4$ and $1 / 4$ to ortho and para states, respectively.

Before presenting the $K_{\mathrm{p}}(T)$ value as computed from eq 10, let us consider the behavior of the dimer partition function $Q_{\mathrm{VR}}^{\mathrm{D}}$ as a function of temperature. Figure 5 represents the 

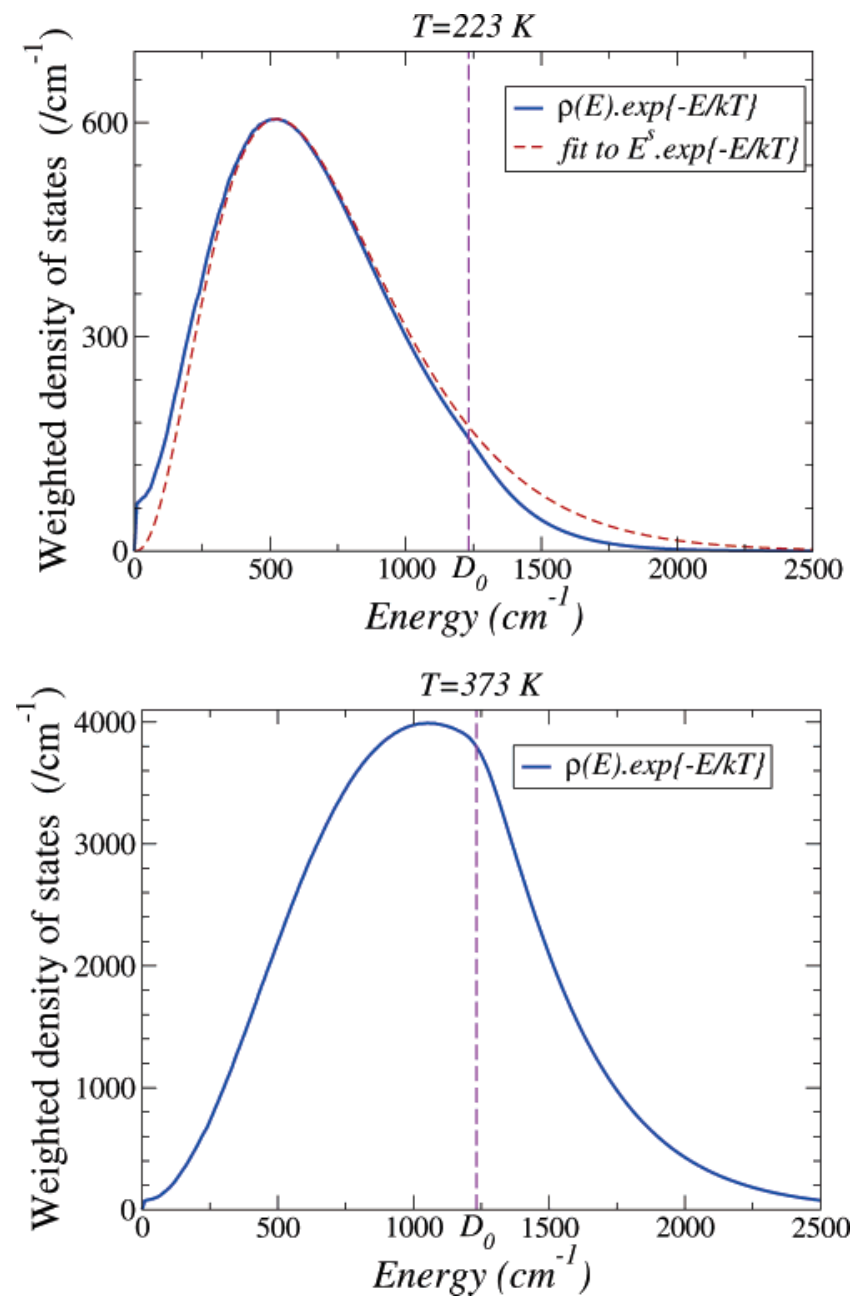

Figure 5. Boltzmann-averaged densities of states $\tilde{\rho}(E, T)=\rho(E) \times$ $\mathrm{e}^{-E / k T}$ (number of rovibrational states per $\mathrm{cm}^{-1}$ ) for temperatures $T=$ 223 and $373 \mathrm{~K}$. The curve at temperature $273 \mathrm{~K}$ can be fitted to the form $\tilde{\rho}(E, T)=A \cdot E^{\mathrm{s}} \cdot \mathrm{e}^{-E / k T}$, where $A=1.05410^{-3}$ and $s=2.52$.

TABLE 1: Symmetry Dependent Factors Accounting for Both Space and Nuclear Spin Degeneracies

$\begin{array}{lllll}\Gamma & \mathrm{A}_{1}^{+} / \mathrm{A}_{1}^{-} & \mathrm{A}_{2}^{+} / \mathrm{A}_{2}^{-} & \mathrm{B}_{2}^{+} / \mathrm{B}_{2}^{-} & \mathrm{E}^{+} / \mathrm{E}^{-} \\ g \Gamma & 1 & 3 & 6 & 3\end{array}$

Boltzmann averaged density of states

$$
\tilde{\rho}(E, T)=\rho(E) \mathrm{e}^{-E / k T}
$$

where $\rho(E)$ corresponds to the standard density of states (i.e., number of rovibrational states per unit energy). The partition function $Q_{\mathrm{VR}}^{\mathrm{D}}$ is related to $\tilde{\rho}(E, T)$ according to

$$
Q_{\mathrm{VR}}^{\mathrm{D}}=\int_{0}^{E_{\max }} \tilde{\rho}(E, T) \mathrm{d} E
$$

Figure 5 shows that, while $\tilde{\rho}(E, T)$ converges for energies below $D_{0}$ at $T \leq 200 \mathrm{~K}$, at higher temperatures there is an increasing fraction of the weighted density extending beyond the dissociation threshold. The occurrence of energies greater than $D_{0}$ can be easily understood within the $J$-shifting approximation. In this approximation, the total energy is defined as the sum of the vibrational and rotational contributions. A $(v$, $J_{\mathrm{o}}, K_{\mathrm{o}}, \Gamma$ ) calculated energy level can thus be dressed by a rotational energy (see eq 8) large enough for the extrapolated energy $E_{v J K}^{\Gamma}$ to lie in the dissociation continuum. This feature is not an artifact of the extrapolation scheme: Such high energy
TABLE 2: Probabilities of the Different $K$ Values Entering the Rovibrational Partition Function for Temperatures 250 and $350 \mathrm{~K}$

\begin{tabular}{cccccccc}
\hline$P(K)$ & $K=0$ & $K=1$ & $K=2$ & $K=3$ & $K=4$ & $K=5$ & $K>5$ \\
\hline$T=250 \mathrm{~K}$ & 0.128 & 0.242 & 0.208 & 0.162 & 0.114 & 0.071 & 0.075 \\
$T=350 \mathrm{~K}$ & 0.118 & 0.224 & 0.198 & 0.161 & 0.120 & 0.081 & 0.098
\end{tabular}

TABLE 3: Comparison between Exact Partial Partition Functions (eq 14) and Results Obtained from Extrapolated Energy Levels and Rotational Constants, for $T=300 \mathbf{K}^{a}$

\begin{tabular}{ccccc}
\hline$Q\left(J=30, K, \mathrm{~A}_{1}^{+}\right)$ & $K=0$ & $K=2$ & $K=4$ & $K=6$ \\
\hline exact & 19.73 & 35.99 & 32.66 & 28.62 \\
approximated & 19.52 & 35.42 & 32.03 & 28.27
\end{tabular}

${ }^{a}$ These values do not take into account the $M$ degeneracy.

levels actually exist and correspond to resonances, i.e., quasibound states, which will decay in the continuum. Many years ago, Smith ${ }^{41}$ discussed how to deal with such states: In the calculation of the partition function, these states should be weighted according to their lifetimes. As these quantities would necessitate enormous calculations for their determination ${ }^{42}$ due to the size of the system considered here, we chose to ignore all quasi-bound states. As a consequence, the calculation of the partition function $Q_{\mathrm{VR}}^{\mathrm{D}}$ will be limited to energy levels $E_{v J K}^{\Gamma}$ located below the dissociation threshold $D_{0}$. We will, however, discuss below the possible influence of these quasi-bound states in the equilibrium constant values. It should also be noted that another type of quasi-bound states exist, which cannot be obtained in our present calculations, namely, Feschbach resonances, where part of the total energy (larger than $D_{0}$ ) is temporarily trapped in modes perpendicular to the dissociation coordinate.

Table 2 displays the probabilities associated to different $K$ values contributing to the partition function for temperatures $T$ $=250$ and $350 \mathrm{~K}$. One can check that $K$ values less than or equal to 5, for which energy levels were actually computed, comprise more than $90 \%$ of the partition function. Conversely, the extrapolated $K$-levels $(K \geq 6)$ comprise less than $10 \%$ of the function.

In the preceding section, the possible effect of centrifugal distortion (a decrease of the $B$ rotational constant with respect to $J$ ) was evoked. To assess this effect, we performed a series of calculations for the value $J=30$, still retaining the symmetric top approximation. Namely, in eq 1 , we replaced the $\mathbf{J}^{2}$ operator by the value $J(J+1)$ (using $J=30$ ) and computed all $\mathrm{A}_{1}^{+}$ bound energy levels for $K$-values $0,2,4$, and 6 . In doing so, we explicitly take into account the centrifugal distortion term $J(J+1) / 2 \mu_{\mathrm{AB}} \mathscr{R}^{2}$. From these energies, one can compute the partial partition functions defined as

$$
Q^{\text {ex. }}\left(J=30, K, \mathrm{~A}_{1}^{+}\right)=\sum_{v} \exp \left(-E_{v, J=30, K}^{\mathrm{A}_{1}^{+}} / k T\right)
$$

(ignoring the $M$ degeneracy). The equivalent approximate quantity $Q^{\text {ap. }}\left(J=30, K, \mathrm{~A}_{1}^{+}\right)$can be obtained from energy levels extrapolated according to eq 8. A comparison of these two sets of values, calculated for $T=300 \mathrm{~K}$, is shown in Table 3 and reveals that the error coming from extrapolated energy levels is on the order of $2 \%$ at most.

B. Equilibrium Constant. Figure 6 presents the calculated equilibrium constant $K_{\mathrm{p}}(T)$ in the range $270-400 \mathrm{~K}$, with and without the contribution of quasi-bound states. In the former case, as the associated lifetimes are not known, these resonance states are affected by weight only determined by the Boltzmann factor $\mathrm{e}^{-E / k T}$. On this figure are also represented the following: 


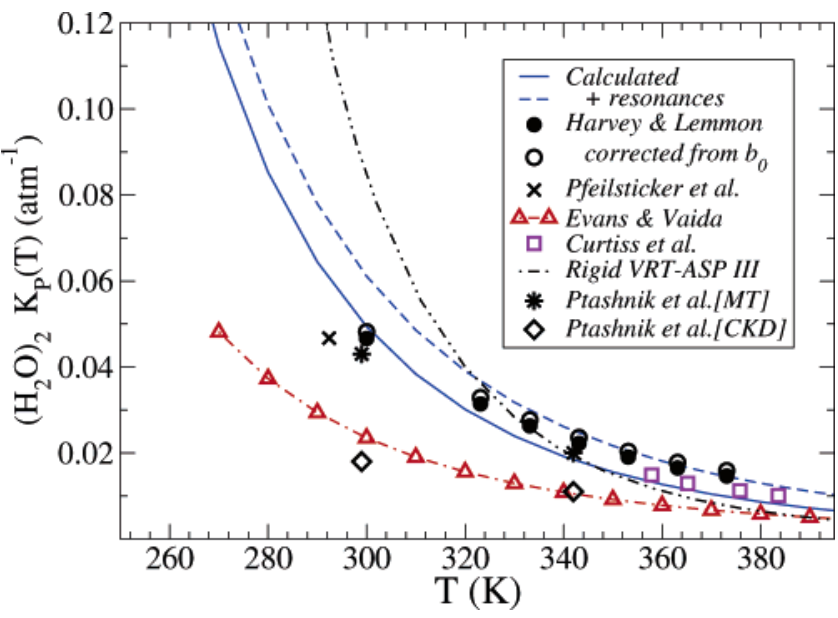

Figure 6. Calculated equilibrium constant $K_{\mathrm{p}}(T)$ in the range 270$400 \mathrm{~K}$, with (- - ) and without ( - ) the contribution of pseudo-bound states and comparison to different experimental data: $(\bullet)$ values $K_{\mathrm{p}}(T)$ $=-\left(B-b_{0}\right) / R T$ extracted from the second virial coefficient data of Harvey and Lemmon, ${ }^{43}$ neglecting the excluded volume $b_{0}$, or $(\bigcirc)$ using the value $38.5 \mathrm{~cm}^{3} / \mathrm{mol}$ as estimated by Curtiss et al.; ${ }^{12}(\mathrm{x})$ direct measurement of dimer concentration in the atmosphere at $T=292 \mathrm{~K}$ by Pfeilsticker et al.; ${ }^{13}(\triangle)$ expression $K_{\mathrm{p}}(T) \simeq 0.0293\left(\mathrm{e}^{\epsilon / k T}-1\right) / T$ $\left(\mathrm{atm}^{-1}\right)\left(\epsilon=D_{0}=3.27 \mathrm{kcal} / \mathrm{mol}\right)$ fitted by Evans and Vaida ${ }^{3}$ to thermochemical data; $(\square)$ results based on the thermal conductivity of steam experiments of Curtiss et al. ${ }^{12}(\cdot-\cdot)$ our previous calculations, ${ }^{18}$ based on the rigid VRT(ASP-W)III potential; estimation by Ptashnik et al. ${ }^{16}$ from high-spectral-resolution pure water vapor absorption spectra using either the CKD-2.4 $(\diamond)$ or Ma and Tipping $(*)$ continuum models.

(i) the results, at high temperatures, based on the thermal conductivity of steam experiments of Curtiss et al, ${ }^{12}$ (ii) the values extracted from the relation ${ }^{12}$

$$
K_{\mathrm{p}}(T)=-\left(B-b_{0}\right) / R T
$$

where $B(T)$ is the second virial coefficient as determined by Harvey and Lemmon $^{43}$ from vaporization data (the above relation was used by either neglecting the excluded volume $b_{0}$ or using the value $38.5 \mathrm{~cm}^{3} / \mathrm{mol}$ as estimated by Curtiss et al. ${ }^{12}$ ), (iii) the direct measurement of dimer concentration in the atmosphere at $T=292 \mathrm{~K}$ by Pfeilsticker et al. ${ }^{13}$ by means of atmospheric long path absorption of the third overtone of the bound $\mathrm{OH}$ stretching mode $\mathrm{OH}_{\mathrm{b}}$, combined with line strength calculated by Low and Kjaergaard, ${ }^{14}$ (iv) the expression

$$
K_{\mathrm{p}}(T) \simeq 0.0293 \frac{\mathrm{e}^{\epsilon / k T}-1}{T}\left(\mathrm{~atm}^{-1}\right)
$$

where $\epsilon=D_{0}=3.27 \mathrm{kcal} / \mathrm{mol}$, fitted by Evans and Vaida ${ }^{3}$ to thermochemical data, (v) our previous results ${ }^{18}$ based on the rigid VRT(ASP-W)III potential, and (vi) the values, at temperatures 299 and $342 \mathrm{~K}$, estimated by Ptashnik et al. ${ }^{16}$ from highspectral-resolution pure water vapor absorption spectra. The two sets of values correspond to using the CKD-2.4 or the Ma and Tipping continuum models, respectively. ${ }^{44}$

One can note a definite improvement in the newly calculated values, with respect to all the experimental data either observed or derived from the second virial coefficient, as compared to our previous calculations. ${ }^{18}$ While it is difficult to ascertain the actual reasons for this, because of many differences in the two sets of calculations, it seems probable that the new extrapolation scheme played a significant role: We explicitly considered all the bound energy levels, up to dissociation, for $J$ values 0,1 , $\ldots, 5(K=0, \ldots, J)$, which allowed us to take into account the
TABLE 4: Rovibrational Partition Functions $Q_{\mathrm{VR}}^{\mathrm{D \Gamma}}\left(\times 10^{-5}\right)$, Excluding the Spin-Degeneracy Factor $g_{\Gamma}$, Associated to the Different IR $\Gamma$ s for the Two Temperatures $T=270$ and 400 K. All Nondegenerate IRs Roughly Contribute to the Same Amount, While the Degenerate Ones $E^{ \pm}$Contribute Twice as Much Because of the Double Occurrence of These Former States (see Figure 1)

\begin{tabular}{ccccccccc}
\hline$Q_{\mathrm{VR}}^{\mathrm{D} \Gamma} \times 10^{-5}$ & $\mathrm{~A}_{1}^{+}$ & $\mathrm{A}_{1}^{-}$ & $\mathrm{A}_{2}^{+}$ & $\mathrm{A}_{2}^{-}$ & $\mathrm{B}_{2}^{+}$ & $\mathrm{B}_{2}^{-}$ & $\mathrm{E}^{+}$ & $\mathrm{E}^{-}$ \\
\hline$T=270 \mathrm{~K}$ & 1.013 & 0.980 & 0.971 & 0.998 & 1.011 & 0.979 & 1.981 & 1.981 \\
$T=400 \mathrm{~K}$ & 3.839 & 3.739 & 3.681 & 3.765 & 3.835 & 3.740 & 7.510 & 7.510
\end{tabular}

energy-level splitting dependence upon the $K$-value. The close agreements between our results and the recent estimation of Ptashnik et al. ${ }^{16}$ comforts their conclusion that water dimer absorption is most probably included partly in the modern CKD continuum model.

C. Approximate Partition Function. When calculating the partition function (eq 11), one can actually restrict the summation to a single IR: Table 4 reveals that, excluding the spin degeneracy factors $g_{\Gamma}$, they all contribute the same amount

$$
Q_{\mathrm{VR}}^{\mathrm{D} \Gamma}(T)=\sum_{v J K} g_{J} \exp \left(-E_{v J K}^{\Gamma} / k T\right)
$$

except for the degenerate species $E^{+}$and $E^{-}$. In fact, Figure 1 shows that $E^{ \pm}$states occur twice as frequently compared to nondegenerate ones. One can thus write the total partition function $Q_{\mathrm{VR}}^{\mathrm{D}}(T)$ according to

$$
Q_{\mathrm{VR}}^{\mathrm{D}}(T) \simeq \frac{32}{16} Q_{\mathrm{VR}}^{\mathrm{DA}_{1}^{+}}(T)
$$

where the factor 32 results from the summation over the spin degeneracies and counting the degenerate species $E^{ \pm}$twice. This property, which was not actually used in the calculations presented here, can reduce by 1 order of magnitude the numerical effort needed to compute the partition function.

D. Thermodynamic Properties. The close agreement between our calculated equilibrium constant $K_{\mathrm{p}}(T)$ and the available experimental data allows us to derive thermodynamical quantities from the partition function. ${ }^{18}$ In doing so, we assume that the dimer behaves like a perfect gas, which is consistent with the way the partition function was calculated (water dimer without any exterior interaction).

The simplest way to estimate the Gibbs free energy for the dimerization process is to use the relation $\Delta G^{\circ}(T)=-R T \ln$ $K^{\circ}(T)$. The dimerization enthalpy $\Delta H^{\circ}(T)$ is calculated from the equation $\Delta H^{\circ}(T)=H_{\mathrm{D}}-2 H_{\mathrm{M}}$ where ${ }^{45}$

$$
H_{\mathrm{X}}=R T\left(\frac{5}{2}+T \frac{\mathrm{d}}{\mathrm{d} T} \ln Q^{\mathrm{X}}\right)
$$

One can then extract the dimerization entropy $\Delta S^{\circ}(T)$ from $\Delta S^{\circ}(T)=\left[\Delta H^{\circ}(T)-\Delta G^{\circ}(T)\right] / T$. Finally, the heat capacity $C_{\mathrm{p}}(T)$ of the dimer is given by

$$
C_{\mathrm{p}}(T)=R\left(\frac{5}{2}+\frac{\mathrm{d}}{\mathrm{d} T} T^{2} \frac{\mathrm{d}}{\mathrm{d} T} \ln Q^{\mathrm{D}}\right)
$$

and $C_{\mathrm{v}}(T)=C_{\mathrm{p}}(T)-R$. The resulting curves are shown in Figure 7 and have been fitted to a quadratic expression $a+b T$ $+c T^{2}$ (valid in the range $190-390 \mathrm{~K}$ ), the coefficients being given in Table 5 .

It can be seen from Figure 7 that the quadratic law is particularly well obeyed by the Gibbs function $\Delta G^{\circ}(T)$, associated to a standard deviation $\sigma=6.78 \mathrm{~J} \cdot \mathrm{mol}^{-1}$. Using the $K_{\mathrm{p}}(T)$ 

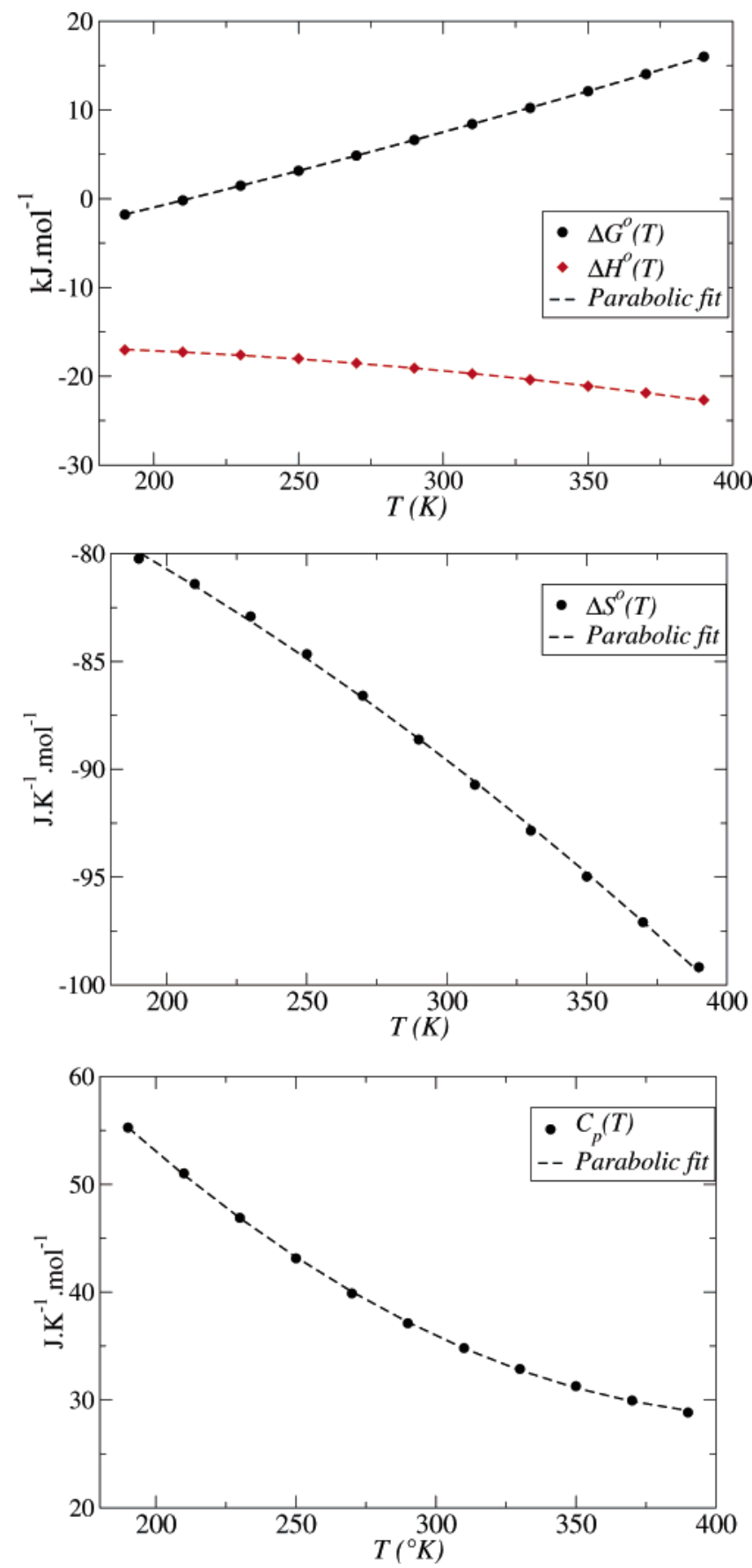

Figure 7. Thermodynamical quantities $\Delta G^{\circ}, \Delta H^{\circ}, \Delta S^{\circ}$, and $C_{\mathrm{p}}$ calculated from the partition function $Q_{\mathrm{D}}$. These curves are fitted to quadratic expressions $a+b T+c T^{2}$, the coefficients being given in Table 5 .

TABLE 5: Quadratic Expression $a+b T+c T^{2}$, Valid in the Range 190-390 K, of the Thermodynamic Quantities

\begin{tabular}{lllr}
\hline & $a$ & \multicolumn{1}{c}{$b$} & \multicolumn{1}{c}{$c$} \\
\hline$\Delta G^{0}\left(\mathrm{~kJ} \cdot \mathrm{mol}^{-1}\right)$ & -14.977 & $6.0065 \times 10^{-2}$ & $4.9618 \times 10^{-5}$ \\
$\Delta H^{0}\left(\mathrm{~kJ} \cdot \mathrm{mol}^{-1}\right)$ & -17.191 & $1.5663 \times 10^{-2}$ & $-7.6546 \times 10^{-2}$ \\
$\Delta S^{0}\left(\mathrm{~J} \cdot \mathrm{K}^{-1} \cdot \mathrm{mol}^{-1}\right)$ & -69.5 & -3.4328 & $-1.0858 \times 10^{-4}$ \\
$C_{\mathrm{p}}\left(\mathrm{J} \cdot \mathrm{K}^{-1} \cdot \mathrm{mol}^{-1}\right)$ & 116.78 & -4.1744 & $4.9342 \times 10^{-4}$
\end{tabular}

$=\exp \left(-\Delta G^{\circ} / R T\right) / p^{\circ}$ relation for the dimerization constant, one can determine the corresponding interpolating formula for this constant

$$
K_{\mathrm{p}}(T)=\mathrm{e}^{-b / R} \exp \left(-\frac{a}{R T}-\frac{c}{R} T\right)\left(\mathrm{atm}^{-1}\right)
$$

Fitting directly our $K_{\mathrm{p}}(T)$ results to this formula leads to the expression

$$
\begin{aligned}
& K_{\mathrm{p}}(T)= \\
& 4.7856 \times 10^{-4} \exp \left(1851.09 / T-5.10485 \times 10^{-3} T\right)\left(\mathrm{atm}^{-1}\right)
\end{aligned}
$$

\section{Discussion}

In this work, we improved upon our previous estimation ${ }^{18}$ of the equilibrium constant $K_{\mathrm{p}}(T)$ by performing more extensive calculations of the bound energy levels: All of them, up to dissociation, were determined for total angular momentum values $J=0, \ldots, 5$ and $K$-values $0, \ldots, J$. Furthermore, we devised an extrapolation scheme to higher $J$-values, which explicitly takes into account the variation with $K$ of both the acceptor and the donor-acceptor tunneling splittings. Also, we employed a flexible potential energy surface, previously fitted to spectroscopical data, which leads to a better description of highly excited states. An interesting extension of our calculations concerns the estimation of thermodynamical quantities and their fitted analytical expressions to a quadratic form over the range 190-390 K. In particular, it is shown that the frequent assumption of isothermicity for $\Delta H^{\circ}$ and $\Delta S^{\circ}$ is not wellsupported by our results.

In any calculation of the dimer partition function, the dissociation energy value $D_{0}$ plays an essential role, as it contributes through the exponential term $\mathrm{e}^{D_{0} / k T}$ which is on the order of $\sim 300$ at room temperature. Consequently, a change of $50 \mathrm{~cm}^{-1}$ in the value of $D_{0}\left(=1234 \mathrm{~cm}^{-1}\right.$ for our flexible potential) results in a change of $26 \%$ of the equilibrium constant $K_{\mathrm{p}}(T)$ at this temperature. Unfortunately, an accurate experimental value for $D_{0}$ does not exist, and all present estimations ${ }^{46}$ contain uncertainties much larger than $50 \mathrm{~cm}^{-1}$. In view of the most recent $a b$ initio calculations performed on the water dimer ${ }^{47}$ one can estimate that our present surface slightly overestimates the $D_{0}$ value, which would result in a small decrease in the calculated $K_{\mathrm{p}}$ values at lower temperature.

One difficult problem encountered in our calculations, and still unresolved, concerns the quantitative role of quasi-bound states or resonances. In principle, the correct way to handle those states has been shown by $\mathrm{Smith}^{41}$ who related the partition function to the collision lifetime matrix in a quantum exact formulation. Similarly, Vigasin ${ }^{48,49}$ has investigated the effect of such resonant states on the collision-induced absorption by means of classical statistical mechanics. It has been argued by Schenter et al. ${ }^{50}$ that such resonances could change the $K_{\mathrm{p}}$ values by as much as 2 orders of magnitude, on the basis of a simplistic water dimer model. Our present results, which bracket the experimental values available depending on whether one takes into account the resonance states or not, show that the effect of such states cannot be so dramatic in the case of the water dimer. One way to estimate this effect would consist of taking into account the lifetimes in an approximate way by means of the microscopic dissociation rate constant $k(E, J)$ such as in the $\mathrm{RRKM}^{51}$ or the statistical adiabatic channel model of Quack and Troe. ${ }^{52,53}$ Indeed, the required ingredients for computing this rate, namely, the density of states associated to some transition-state geometry $\mathscr{R}^{*}$, could easily be obtained within our formulation. To conclude this discussion on the possible role of resonance states, it should be kept in mind that their effects are designed to be limited in the temperature range 200$300 \mathrm{~K}$ (see Figure 5), which is the one relevant to atmospheric processes. 
Acknowledgment. This work was partially funded by a CNRS-NSF grant. The Berkeley effort is supported by Experimental Physical Chemistry Program of the National Science Foundation.

\section{References and Notes}

(1) Daniel, J. S.; Solomon, S.; Sanders, R. W.; Portman, R. W.; Miller, D. C.; Madsen, W. J. J. Geophys. Res. [Atmos.] 1999, 104, 16785.

(2) Chylek, P.; Geldart, D. J. Geophys. Res. Lett. 1997, 24, 2015.

(3) Evans, G. T.; Vaida, V. J. Chem. Phys. 2000, 113, 6652.

(4) Vaida, V.; Daniel, J. S.; Kjaergaard, H. G.; Goss, L. M.; Tuck, A.

F. Q. J. R. Meteorol. Soc. 2001, 1627.

(5) Vaida, V.; Kjaergaard, H. G.; Feierabend, K. J. Int. Rev. Phys. Chem. 2003, 22, 203.

(6) Devir, A. D.; Neumann, M.; Lipson, S. G.; Oppenheim, U. P. Opt. Eng. 1994, 33, 746

(7) Daniel, J. S.; Solomon, S.; Kjaergaard, H. G.; Schofield, D. P. Geophys. Res. Lett. 2004, 31.

(8) Pruppacher, H. R.; Klett, J. D. Microphysics of Clouds and Precipitation; Reidel: Norwell, MA, 1978

(9) Schenter, G. K.; Kathmann, S. M.; Garett, B. C. Phys. Rev. Lett. 1999, 82,3484 8874

10) Loerting, T.; Liedl, K. R. Proc. Natl. Acad. Sci. U.S.A. 2000, 97

(11) Aloiso, S.; Francisco, J. S.; Friedl, R. R. J. Phys. Chem. A 2000, 104,6597

(12) Curtiss, L. A.; Frurip, D. J.; Blander, M. J. Chem. Phys. 1979, 71 2703.

(13) Pfeilsticker, K.; Lotter, A.; Peters, C.; Bösch, H. Science 2003 , $300,2078$.

(14) Low, G. R.; Kjaergaard, H. G. J. Chem. Phys. 1999, 110, 9104

(15) Kassi, S.; Macko, P.; Naumenko, O.; Campargue, A. Phys. Chem. Chem. Phys. 2005, 7, 2460

(16) Ptashnik, I. V.; Smith, K. M.; Shine, K. P.; Newnham, D. A. Q. J. R. Meteorol. Soc. 2004, 2391.

(17) Goldman, N.; Fellers, R. S.; Leforestier, C.; Saykally, R. J. J. Phys. Chem. A 2001, 105, 515.

(18) Goldman, N.; Leforestier, C.; Saykally, R. J. J. Phys. Chem. A 2004 108, 787.

(19) Leforestier, C.; Gatti, F.; Fellers, R. S.; Saykally, R. J. J. Chem. Phys. 2002, 117, 8710

(20) Brocks, G.; an der Avoird, A.; Sutcliffe, B. T.; Tennyson, J. Mol. Phys. 1983, 50, 1025 .

(21) an der Avoird, A.; Wormer, P. E. S.; Moszynski, R. Chem. Rev. 1994, 94, 1931

(22) Baciæ, Z.; Light, J. C. J. Chem. Phys. 1986, 85, 4594.

(23) Baciæ, Z.; Light, J. C. J. Chem. Phys. 1987, 87, 4008.

(24) Leforestier, C.; Braly, L. B.; Liu, K.; Elrod, M. J.; Saykally, R. J. J. Chem. Phys. 1997, 106, 8527.
(25) Fellers, R. S.; Leforestier, C.; Braly, L. B.; Brown, M. G.; Saykally, R. J. J. Chem. Phys. 1999, 110, 6306

(26) Dyke, T. R. J. Chem. Phys. 1977, 66, 492

(27) Lanczos, C. J. Res. Natl. Bur. Stand. (U.S.) 1950, 45, 255.

(28) Cullum, J. K.; Willoughby, R. A. Lanczos Algorithms for Large Symmetric Eigenvalues Computations; Birkhäuser: Boston, 1985.

(29) Wyatt, R. E.; Scott, D. S. Mathematical Studies Series; Cullum, J. K., Willoughby, R. A., Eds.; North-Holland: Amsterdam, 1986; Vol. 127. (30) Smith, S. C.; Yu, H.-G.; Leforestier, C.; Rayez, J. C. Phys. Chem. Chem. Phys. 1999, 1, 1311.

(31) Polyansky, O. L.; Jensen, P.; Tennyson, J. J. Chem. Phys. 1996 105,6490

(32) Xantheas, S. S. J. Chem. Phys. 1996, 104, 8821.

(33) Schütz, M.; Brdarski, S.; Widmark, P.-O.; Lindh, R.; Karlström, G. J. Chem. Phys. 1997, 107, 4597.

(34) an Duijeneveldt van de Ridjt, J. G. C. M.; van Duijeneveldt, F. B.

J. Chem. Phys. 1999, 111, 3812.

(35) Klopper, W.; Lüthi, H. P. Mol. Phys. 1999, 96, 559.

(36) Harris, D. O.; Engerholm, G. G.; Gwinn, W. D. J. Chem. Phys. $\mathbf{1 9 6 5}, 43,1515$.

(37) Echave, J.; Clary, D. C. Chem. Phys. Lett. 1992, 190, 225.

(38) Bowman, J. M. Adv. Chem. Phys. 1985, 61, 115.

(39) Mielke, S. L.; Lynch, G. C.; Truhlar, D. G.; Schwenke, D. W. Chem. Phys. Lett. 1993, 216, 441.

(40) Harris, G. J.; Viti, S.; Mussa, H. Y.; Tennyson, J. J. Chem. Phys. 1998, 109, 7197.

(41) Smith, F. T. J. Chem. Phys. 1962, 38, 1304.

(42) Moiseyev, N. Phys. Rep. 1998, 302, 211.

(43) Harvey, A. H.; Lemmon, E. W. J. Phys. Chem. Ref. Data 2004 33,369 .

(44) Kjaergaard, H. G.; Robinson, T. W.; Howard, D. L.; Daniel, J. S.; Headrick, J. E.; Vaida, V. J. Phys. Chem. A 2003, 107, 10680.

(45) Mayer, J. E.; Mayer, M. G. Statistical Mechanics, 2nd ed.; WileyInterscience: New York, 1977.

(46) Klopper, W.; van Duijeneveldt van de Ridjt, J. G. C. M.; van Duijeneveldt, F. B. Phys. Chem. Chem. Phys. 2000, 2, 2227.

(47) Tschumper, G. S.; Leininger, M. L.; Hoffman, B. C.; Valeev, E. F.; Schaefer, H. F.; Quack, M. J. Chem. Phys. 2002, 116, 690 (48)

(48) Vigasin, A. A. J. Quant. Spectrosc. Radiat. Transfer 1995, 56,

(49) Vigasin, A. A. Molecular Complexes in Earth's, Planetary, Cometary, and Interstellar Atmospheres; Vigasin, A. A., Slanina, Z., Eds.; World Scientific Publishing: Singapore, 1998.

(50) Schenter, G. K.; Kathmann, S. M.; Garett, B. C. J. Phys. Chem. A 2002, 106, 1557.

(51) Gilbert, R. G.; Smith, S. C. Theory of Unimolecular and Recombination Reactions; Blackwell Scientific Publications: Oxford, 1990.

(52) Quack, M.; Troe, J. Int. Rev. Phys. Chem. 1981, 1, 97.

(53) Quack, M.; Troe, J. Theoretical Chemistry, Advances and Perpectives; Henderson, D., Ed.; Academic Press: New York, 1981; Vol. 6B. 